\title{
OPTIMIZATION OF SERVICE ROUTES IN A RESTRICTED ENVIRONMENT
}

\author{
Stephen F. Bashford, Research Assistant \\ William H. Askew, Lecturer \\ University of Nottingham \\ Department of Civil Engineering \\ University Park \\ Nottingham NG7 2RD \\ United Kingdom
}

\begin{abstract}
This paper presents a method which is presently being developed for planning mechanical and electrical service routes within a confined space. The method uses a modified Lees' algorithm which allows horizontal, vertical and diagonal $\left(45^{\circ}\right)$ routings within the space. Each service is initially routed individually and then a system based on simulated evolution techniques is used to select services for progressive re-routing until an optimal solution is obtained. The method of selecting which services are to be re-routed considers the length and complexity of the service route, whether its initial routing clashes with any other services and whether its position will permit a logical construction sequence. The order in which the services are re-routed is then determined by a probabilistic method. Initial tests using this method indicate that complex service layouts are successfully routed and that the resultant layouts are well proportioned and distributed.
\end{abstract}

\section{INTRODUCTION}

Various studies have been undertaken to determine which construction activities would most benefit from some form of automation or robotization and which activities may be most feasible to automate. Whilst these studies have not reached a definitive solution $[1,2,3]$ there is general agreement that the success of implementing a robotics system is largely dependent upon partly altering construction methods 'to match the requirements for automation' [4].

Nottingham University was asked by a major UK contractor to examine the work undertaken by the company with the aim of determining which activities should be considered for some form of automation or robotization. In a multi round Delphi [5] study, 120 of the company's site managers were questioned about general site activities and their associated problems; the problems and benefits of robotic systems in construction; and the preferred activities for robotization. It was subsequently determined that service layout and design was an activity that causes particular problems on site [6]. The 4 major reasons for this were: 
- lack of design co-ordination,

- drawing detail problems,

- late delivery of parts,

- lack of coordination of site activities.

However, service installation was also shown to be an activity which the participants did not believe could be easily automated, primarily for the reasons listed above. Consequently, whilst there is considerable recognition, by both the survey participants and the industry in general $[1,2,6,7]$ that this activity is a key cause of delay, there is considerable doubt that this activity could be automated without first improving the management and control of its design $[6,8]$.

This paper describes the method being developed, at Nottingham University, for automatically planning the mechanical and electrical service route layouts in confined spaces where clashes between the routes are likely to occur. Once a successful automated service layout design and planning package has been developed it could then be linked to an automated fabrication and assembly system to form an integrated management, design and construction package $[2,3,9]$.

\section{GENERAL DESCRIPTION OF METHOD}

The key to designing a good service route layout is to ensure that the various services do not clash, that they follow as short a path as possible (whilst attempting to ensure that this does not require other services to follow excessively long paths), that they avoid any fixed obstructions within the routing space and that the total 'cost' of the system is minimized. The resultant routes should then be clearly presented in drawings which represent both the individual service and the total routing region. Furthermore, the service design package should also be able to prepare tables of inventory requirements for each service.

The method uses the following basic procedure for routing the services:

1. The dimensions of the routing space, including any projections, such as columns and beams, transformers etc. are supplied and converted into grid dimensions which are then used to mark occupied areas in a three dimensional (3D) array, based upon a $50 \mathrm{~mm}$ grid, which represents the routing space.

2. Key fixed coordinates for each of the dimensioned services to be routed are supplied and transferred to the 3D occupancy array. These generally represent points where the service enters the routing space or points where there are outlets or connections.

3. Using a modified Lees' [10] algorithm combined with a 'minimum path length' search routine for branching services, each service is routed, without considering clashes with other services, in order to find the shortest connection distance. 
4. The cost of each service is then determined to assist in selecting which services are to be re-routed.

5. The services selected are then re-routed. During this re-routing those services that were not selected or which have already been re-layed may be cut by the service being routed, though this will incur a cost penalty.

6 . The cost of each service is then determined and those services selected for rerouting are removed and placed in a queue in accordance with the order required by the simulated evolution technique [11,12].

7. The procedure outlined in $5 \& 6$ is repeated until a solution is obtained, which does not contain service clashes and where the total routing cost is within a pre-defined limit of the optimum cost possible for each service and the routing region as a whole.

8. Once a successful solution has been obtained the resultant coordinates for the solution may then be linked to a CAD package for presentation.

The important feature of this service routing method are that:

1. Any number of layers are permissible.

2. Orthogonal (manhattan) and $45^{\circ}$ routings [13] are allowed.

3. Pre-defined obstructions and routed services are allowed.

4. The dimensions of the service and its minimum turning radius are considered in the routing.

5. Preferred zones of installation are allowed for each service to reflect the order of construction.

In the remainder of the paper the process of routing individual and branching services is described and the method of selecting services for re-routing is detailed. In addition some indication of the present level of development of the system and its success is provided.

\section{ROUTING OF SINGLE SERVICES}

The method used for routing the services is based on simulated evolution techniques applied to a modified Lees' algorithm. Traditionally Lees' algorithms [10] have only been used for manhattan style routing in a pre-defined three-dimensional rectangular grid, however the algorithm can be modified to also allow routings at $45^{\circ}$. Even with this modification (a) the algorithm will always find a path if one exists, and (b) the path the algorithm finds will always have the minimum possible cost [14].

The procedure for the modified Lees' algorithm is as follows (see figure 1, showing a simplified two dimensional routing): 
1. For each admissible neighbour of the cell at grid reference ' $S$ ' which has a current occupancy value ' $\mathrm{V}$ ', assign a value ' $\mathrm{V} 1^{\prime}$ ' which is calculated by :

$\mathrm{V} 1$ = length of route + penalty value of destination cell

2. Repeat the process for each of the admissible cells adjoining cell ' $S$ ' using the value ' $\mathrm{V} 1^{\prime}$ ' as the new occupancy value ' $\mathrm{V}$ ' for that cell.

3. If a cell is already occupied by a value ' $\mathrm{V}$ ' overwrite the existing value if the alternative value ' $\mathrm{V} 1$ ' is smaller.

4. Repeat this procedure of assigning the lowest occupancy value ' $\mathrm{V} 1$ ' to each vacant cell adjoining each occupied cell until the goal ' $F$ ' is reached.

5. To trace the shortest path start from the goal and search for the adjoining cell with the smallest occupancy value, from that cell repeat the process until the origin is reached.

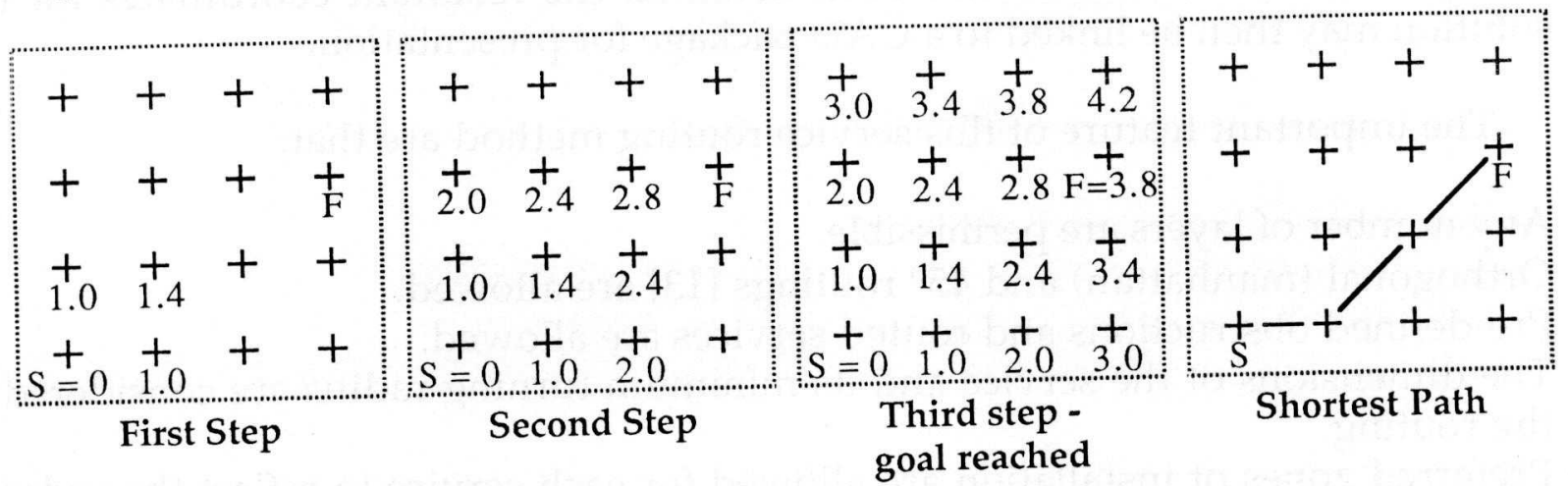

\section{Modified Lees' Algorithm Procedure}

Figure 1.

The cost of a particular path may be defined in any manner. In this routing system the total cost of a service routing reflects (a) the length of the routing, (b) the number of changes of direction within the routing, (c) penalties for cutting other service routes, and (d) penalties for leaving the preferred routing zone for that service. The appropriate values attached to the penalties are presently being examined and will reflect (a) the difficulties of routing the service under consideration, (b) the cost of the service and (c) the size of the existing services.



Empty Grid (with penalties)



Completed grid

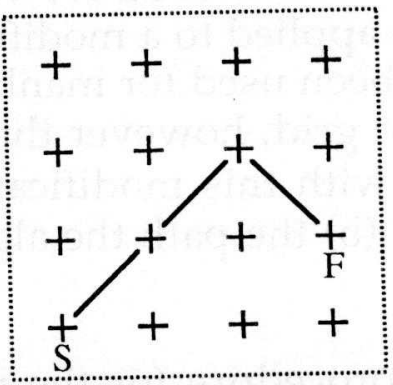

Path with least connection cost

Modified Lees' Algorithm with Penalty Values

Figure 2 
In figure 2, two of the cells have penalties ' $\mathrm{P}$ ' attached to them, which represent a service already passing through these positions. Consequently the shortest path is not the most direct path as higher costs are associated with cutting the existing service than using an alternative route.

\section{ROUTING OF BRANCHING SERVICES}

The major problem with the modified Lees' algorithm is that it will always find the shortest path from a given starting point ' $\mathrm{S}$ ' to each finishing point ' $\mathrm{F}$ '. Hence, whilst the individual routes will be the shortest distance possible it is probable that a combined route serving several finishing points will be shorter. In order to find the shortest distance to connect multiple points a Steiner Tree [15] would need to be used. However, finding the optimum Steiner distance is a complex process which would be made even more difficult by the restrictions imposed by the grid structure of the modified Lees' algorithm and the obstructions that may prevent direct routing between points [16] (see figure 3).

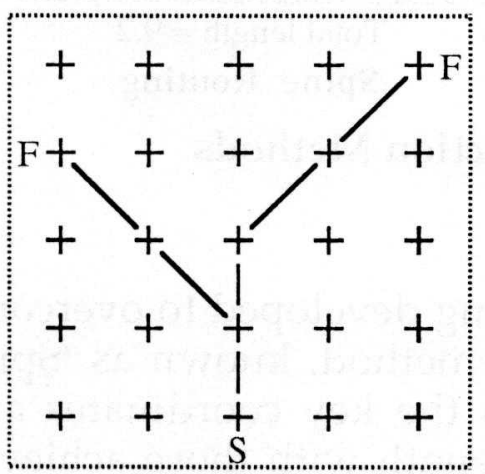

Total length $=7.6$

Lees' Routing

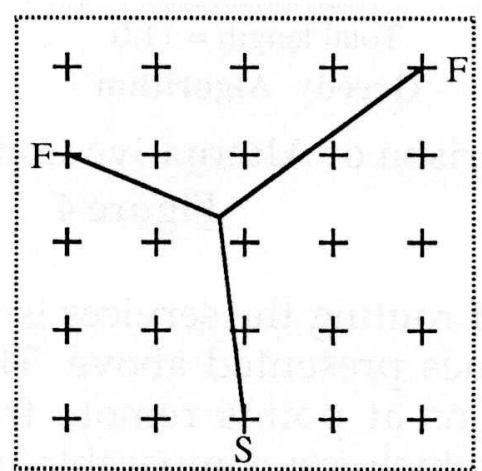

Total length $=7.01$

Steiner Routing

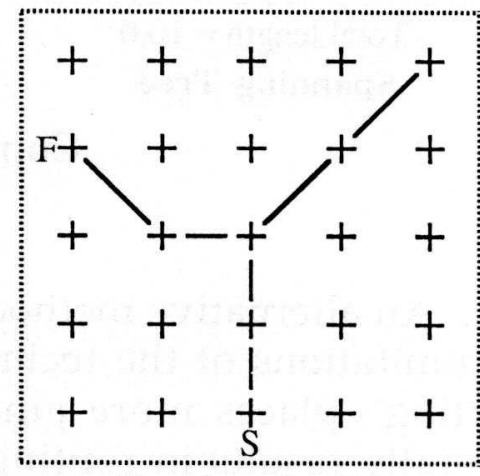

Total length $=7.2$

Quasi-Steiner Routing

Comparison of Connection Distances Between Lees' and Steiner Routings Figure 3

The two most common methods for finding near optimum solutions for connecting multiple points are:

1. Spanning Tree - in this method the two key points linked by the shortest distance (or modified Lees' distance) are joined and progressively the remaining points are connected to these using the next available shortest connection distance. The connections are checked to ensure that no loops are created. It has been shown that connecting using this method will never result in a total connection distance more than $15.39 \%$ longer than that achieved using a Steiner tree. However, this method will result in most junctions occurring at key points which could cause undue congestion when routing services $[15,16]$. 
2. Greedy Algorithm - in this method routing for each service proceeds from left to right across the routing space from each key point and involves routing one grid column at a time. If a key point in a given column is occupied by the same service as the intercepting row then they are connected, otherwise the contents of the row are extended to the next column where the process is repeated. This method is not easily applied to examining diagonal routes and is also complex to use when the routing area is partly obstructed. However, it does ensure that fewer interceptions occur at the key points [12] .

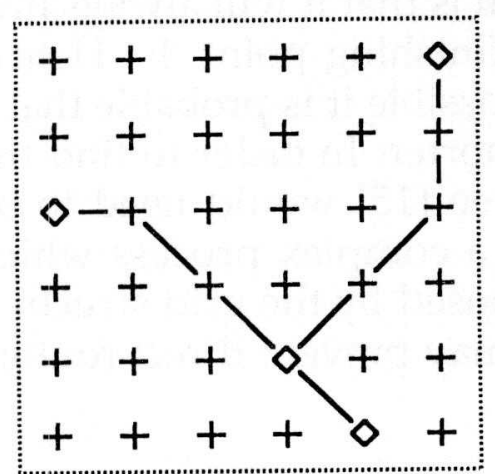

Total length $=10.0$

Spanning Tree

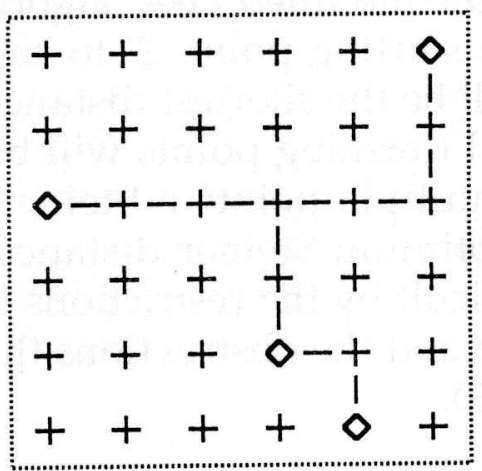

Total length $=11.0$

Greedy Algorithm

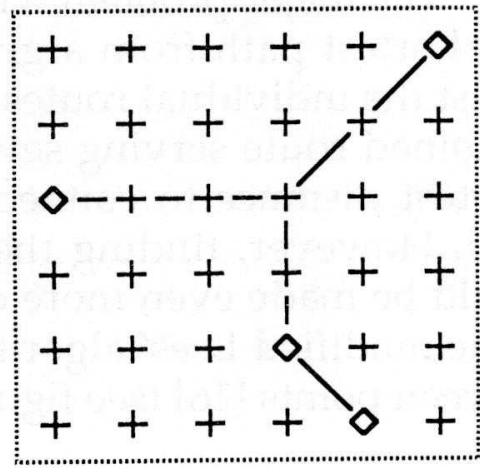

Total length $=9.2$

Spine Routing

\section{Comparison of Alternative Connection Methods}

Figure 4

An alternative method of routing the services is being developed to overcome the limitations of the techniques presented above. This method, known as 'Spine Routing', places more junctions at points remote from the key coordinates and generally results in routings which are comparable in length with those achieved using the spanning tree technique. In this method the two key points connected by the longest Lees' path are joined to form the spine of the services layout. The key point nearest to the spine is then connected by the shortest modified Lees' path. Progressively the remaining points are connected in the same manner to either the spine or other points which are already connected to the spine.

The initial tests using the spine method indicate that layouts created using this technique will be more economic than those which use the spanning method and will result in a less congested and simpler solution (see section VI).

\section{SELECTION OF SERVICES FOR RE-ROUTING}

Following the initial individual routing of the services to determine their minimum connection cost, the services are each re-routed in order of cost, with the most costly services being routed first. During this re-routing each service being placed will find the most economic route available to connect each of its key points by using the modified Lees' and Spine routing techniques. This necessitates routing 
around fixed features of the routing space and services already placed, or cutting services where this provides a more economic path. After completion of the rerouting of all the services the cost of each service can be recalculated by summing the associated weighted values (see section 3 ).

A technique based upon artificial evolution is then used to select which services are to be re-routed, which ensures that the solution does not become trapped in a local minimum, as even services which are near optimum may be re-routed $[11,12]$. The basis of this method is that each service of the current generation is scored for its 'goodness' by normalizing the cost of the service, in comparison with its optimum routing cost, within a range of 0.0 to 1.0. Its chances of surviving into the next generation (iteration) then depends on its 'goodness' being greater than a defined survival threshold value. This threshold value is determined by producing a randomly generated number in the range 0.0 to 1.0. Those services which did not pass the current 'goodness' threshold are then removed and placed in a queue for rerouting. The order of re-routing places the worst service first and re-routes it using the techniques described in sections III and IV .

An additional mutation mechanism is also used to ensure that services with high routing costs (which will generally be routed on an optimal path and are consequently less likely to be subject to re-routing), are on occasion re-routed despite their having passed the current 'goodness' threshold. The method operates by randomly selecting a service and then multiplying its current cost by a randomly generated number and then comparing the resultant figure to the services optimal cost. If the new normalized cost then fails to pass the current 'goodness' threshold the service is place in the appropriate position in the queue for re-routing.

The frequency with which this mechanism is applied must allow convergence on a solution to occur whilst preventing a repetitious sequence of re-routing developing. This will ensure that different optimal solutions are investigated. It will also prevent rapid convergence upon the same solution, which may only represent a local minimum, rather than a general optimal solution.

This mechanism is comparable to the evolutionary theory of Punctuated Equilibria $[17,18]$ which states that a powerful method of causing change once statis has developed in a population is to introduce existing species into a new environment. Or in this case to force a routing solution which has reached statis into a new path by the deliberate manipulation of its re-routing selection mechanism.

Each iteration of this re-routing process will resolve some violations while, possibly, creating new violations. The iterative process continues until no violations remain [12] and the total cost is within a defined limit of the optimum cost possible for each service and the routing region as a whole. 


\section{PRELIMINARY RESULTS \& FUTURE WORK}

The routing program has been written in Turbo Pascal, version 6.0, and is currently undergoing evaluation. The aim of the preliminary tests is to determine the appropriate weighting values associated with (a) the costing of the service routes, (b) the termination criteria, and (c) the rate of mutation. In addition the efficiency of the spine routing method is also to be examined.

\begin{tabular}{|c|c|c|c|c|c|}
\cline { 2 - 5 } \multicolumn{1}{c|}{} & $\begin{array}{c}\text { Average } \\
\text { length } \\
\text { of route }\end{array}$ & \multirow{2}{*}{$\begin{array}{c}\text { SD } \\
\sigma \mathrm{n}-1\end{array}$} & \multicolumn{2}{|c|}{ Average no. of junctions at: } & $\begin{array}{c}\text { Average } \\
\text { number of } \\
\text { corners }\end{array}$ \\
\cline { 4 - 5 } Spanning Tree & 12.01 & 3.97 & 212 & 0 & 1.92 \\
\hline Spine method & 12.21 & 4.05 & 82 & 130 & 0.96 \\
\hline
\end{tabular}

Summary of test results comparing spanning and spine routing Table 1

The spine routing method has been compared with the spanning tree for 104 groups of $3,4,5$ or 6 randomly placed key coordinates (in a ten by ten, two dimensional grid). The results, which are illustrated in Table 1, show that the spine method compares favourably with the spanning tree. Whilst the overall routing length was on average $1.7 \%$ longer using the spine method the number of corners (not associated with junctions) was 50\% less, providing a considerable cost saving. Furthermore, the spanning method concentrates all the junctions at the key coordinate positions whilst the spine method distributes them more evenly over the routing zone. This test is to be extended to include three dimensions, obstructions and up to 10 sets of key coordinates.

Presently the appropriate values for the termination criteria are being examined and initial results indicate that for layouts with a high density of services (more than $50 \%$ of the volume occupied) that a total connection cost of 1.33 times the sum of the optimum individual service route costs may be appropriate. However, these tests are based upon small routing areas containing non-branching services which may not be comparable to complex layouts.

In addition to the preliminary tests to evaluate the appropriate weighting values the method will also being compared to manually routed service layouts and standard problems that have been developed for evaluating the efficiency of electrical switchbox routers $[12,16,18]$.

\section{CONCLUSION}

The method presented in this paper for routing services in a confined and complex area appears to offer a flexible and efficient routing mechanism. Service 
layouts designed by this method are coordinated and practical. Furthermore, as construction proceeds the method will enable rapid re-designs of the layout to reflect:

1. different user requirements,

2. changes in the order of construction,

3. the introduction of new fixed features,

4. the introduction of new key coordinate positions.

By using this method the efficiency of service installation will be increased; resulting in immediate savings to the industry as the existing labour will be able to operate more efficiently and materials wastage will be reduced. Ultimately, an integrated design and construction system $[2,19]$ may be developed which utilizes the design coordination information of this service routing technique to control the operations of automated fabrication and installation robots.

\section{ACKNOWLEDGEMENTS}

The authors would also like to thank M.J.Mawdesley of the Department of Civil Engineering at Nottingham University for his advice in preparing this paper. In addition the financial and technical support of Tarmac Construction Ltd has been invaluable.

\section{REFERENCES}

[1] Kangari R \& Halpin D.W, "Potential Robotics Utilization in Construction", in Journal of Construction Engineering and Management, ASCE.CO.Vol 115(1), Pg 126 - 143, 1988.

[2] Tucker R.L, "High Payoff Areas for Automation Applications", in Proc. 5th International Symposium of Robotics in Construction, Tokyo, Japan, Pg 9 -16, June 6 - 8, 1988.

[3] Tatum C, "Design and Construction Automation: Competitive Advantages and Management Challenge", in Proc. 6th International Symposium of Robotics in Construction, San Francisco, USA, Pg 332 - 339, June 6 - 8, 1989.

[4] Atkin B, Atkinson P, Bridgewater C \& Ibanez-Guzman J, "A New Direction in Automating Construction", in Proc. 6th International Symposium of Robotics in Construction, San Francisco, USA, Pg 119 - 126, June 6 - 8, 1989.

[5] Linstone H.A \& Turoff M, "The Delphi Method - Techniques and Applications", Addison - Wesley Publishing Company, USA, 1975.

[6] Bashford S.F \& Askew W.H, "Initial Task Selection for Detailed Robotic Evaluation", in Proc. 9th International Symposium of Robotics in Construction, Tokyo, Japan, 1992.

Fisher D.J \& O'Connor J.T, "Piping Erection Constructability Issues in a Semi-automated Environment", in Proc. 6th International Symposium of Robotics in Construction, San Francisco, USA, Pg 167 - 174, June 6 - 8, 1989. the UK construction industry", in Proc. 6th International Symposium of Robotics in Construction, San Francisco, USA, Pg 103 - 110, June 6 - 8, 1989. 
[9] Koskela L \& Salagnac J.L, “Determinants of the Development of Computer Integrated Construction", in Proc. 7th International Symposium of Robotics in Construction, Bristol, England, Pg 245 - 252, June 5 - 7, 1990.

[10] Lee.C.Y, "An Algorithm for Path Connections and its Application", IRE Trans. Electronic Computation, Vol. EC10, Pg 346-365, 1961.

[11] Kling R.M \& Banerjee P, "ESP: A new standard cell placement package using simulated evolution", in Proc. 24th Design Automation Conference, Pg 60 - 66, 1987.

[12] Lin Y, Hsu Y \& Tsai F, "SILK: A Simulated Evolution Router", in IEEE Trans. on Computer Aided Design, Vol. 8, No. 10, October 1989.

[13] Clegg P, "PC-based autorouting : an evaluation", in CAD, Vol. 22, No. 10, December 1990.

[14] Rubin F, "The Lee Path Connection Algorithm", in IEEE Trans. on Computers, Vol. C-23, No. 9, September 1974.

[15] Stewart I,"Trees,telephones and tiles",in New Scientist, Pg 26-29,16 Nov 1991.

[16] Gerez S.H \& Herrmann O.E, "Switchbox Routing by Stepwise Reshaping", in IEEE Trans. on Computer Aided Design, Vol. 8, No. 12, December 1989.

[17] Dawkins R, "The Blind Watchmaker", Longman Scientific and Technical Press, UK,1986.

[18] Cohoon J.P, Hegde S.U, Martin W.N \& Richards D, "Floorplan Design Using Distributed Genetic Algorithms", in IEEE Trans. on Computers, 1988.

[19] Tucker R.L, "Construction Automation in the United States", in Proc. 7th International Symposium of Robotics in Construction, Bristol, England, key note speech, June $5-7$, 1990. 